schlechteren Prognose assoziiert. Die Gesamtsterblichkeit sei nach den Ergebnissen der ADVANCE-Studie nach Adjustierung um $61 \%$ erhöht. Daraus ergebe sich die Notwendigkeit, Vorhofflimmern zu verhindern bzw. konsequent zu behandeln.

\section{Erhöhtes Risiko für den akuten Herztod}

Diabetiker tragen auch ein erhöhtes Risiko für den akuten Herztod. Besonders betroffen sind solche mit einer Herzinsuffizienz oder Zustand nach Myokardinfarkt. „Der entscheidende Risikofaktor neben der KHK ist die autonome Neuropathie“, so Dill. Deshalb sollte bei allen Diabetikern regelmäßig ein 12-KanalEKG abgeleitet werden, wobei das besondere Augenmerk auf die QT-Zeit gerichtet sein sollte. Darüberhinaus gilt es, mittels Belastungsuntersuchung nach einer KHK zu fahnden. Bei diabetischen Patienten mit unklarer Synkope und struktureller Herzerkrankung kann auch eine elektrophysiologische Untersuchung mit programmierter Ventrikelstimulation hilfreich sein.
HERZLICH WILLKOMMEN DIABETES HERE STTAGUNG

11. - 12. NOVEN BER 2016, NÜRNB :RG

Da auch Hypoglykämien einen akuten Herztod auslösen können, sollten bevorzugt antidiabetische Strategien eingesetzt werden, die das Hypoglykämierisiko nicht erhöhen.

Dr. med. Peter Stiefelhagen

Quelle: Symposium „Diabetes, Herz und Gefäße - Koronare Herzkrankheit” Herbsttagung der Deutschen Diabetes Gesellschaft (DDG), am 11.11.2016 in Nürnberg

\title{
GLP-1-Analoga auch für Typ-1-Diabetiker?
}

\section{GLP-1-Analoga sind eine bewährte Option bei Typ- 2-Diabetikern. Doch sollten sie auch bei übergewich- tigen Typ-1-Diabetikern erwogen werden? Das Wirkprinzip ist ähnlich wie bei Typ-2-Diabetikern, aber das Hypoglykämierisiko erhöht.}

GLP-1-Analoga verbessern den $\mathrm{HbA}_{1 \mathrm{c}}$-Wert bei Typ-2-Diabetikern um ca. 1,5\%. „Dabei sind verschiedene lokale und systemische Effekte beteiligt“, so Dr. Dominik Bergis von der medizinischen Universitätsklinik in Frankfurt a. M. Dazu gehören die Stimulation der glukoseabhängigen Insulinsekretion, die Hemmung der Glukagonsekretion, die Verlangsamung der Magenentleerung und die Steigerung des Sättigungseffektes durch eine zentralnervöse Wirkkomponente.

Die Einstellung eines Typ-1-Diabetikers ist nicht immer einfach; denn erhöhte Glukagon- und erniedrigte Amylin-Spiegel begünstigen eine Hyperglykämie. Außerdem kommt es unter der Insulintherapie häufig zu einer Gewichtszunahme und es besteht ein Hypoglykämierisiko. Belastend für viele Patienten sind auch die vielen Injektionen. „Doch es gibt bisher keine Alternative zur Insulintherapie, denn GLP-1-Analoga sind für diese Indikation nicht zugelassen", so Bergis. Anforderungen, die an eine solche Alternative gestellt werden müssten, sind die Unterdrückung der Glukagonsekretion während einer Mahlzeit, eine Wirksamkeit auch bei residueller oder fehlender Betazellfunktion, ein positiver Effekt auf die Betazellmasse, eine Gewichtsabnahme oder zumindest eine Stabilisierung des Körpergewichts und keine Erhöhung des Hypoglykämierisikos.

\section{Gleiche Wirkungen wie bei Typ-2-Diabetkern}

Dass die GLP-1-Achse auch bei Typ-1-Diabetikern funktioniert, dies konnte in entsprechenden Studien zweifelsfrei belegt werden, d.h. nach endogenem oder exogenem GLP-1 kommt es zu einer antidiabetischen Wirkung, und zwar unabhängig davon, ob noch eine residuelle Betazellfunktion besteht. Auch führte die Gabe von Exenatid oder Sitagliptin zusätzlich zu Insulin zu einer Abnahme des Körpergewichts unter dem GLP-1-Analogon, einem geringeren Bedarf an Insulin unter beiden inkretinbasierten Therapiestrategien und zu einer stärkeren $\mathrm{HbA}_{1 \mathrm{c}}-\mathrm{Sen}$ kung im Vergleich zur Monotherapie mit Insulinen. Bei gleich guter Stoffwechseleinstellung führte auch Liraglutid zu einer signifikanten Abnahme der notwendigen Insulindosis und zwar unabhängig davon, ob noch eine Restfunktion der Betazellen vorhanden war oder nicht. Diese Effekte einer zusätzlichen Liraglutid-Gabe auf den $\mathrm{HbA}_{1 \mathrm{c}}$-Wert, die erforderliche Insulindosis und das Körpergewicht konnten dann auch in zwei Studien (ADJUNCT ONE und ADJUNCT TWO) nachgewiesen werden, wobei die Wirkung dosisabhängig war. Allerdings stieg die Rate an symptomatischen Hypoglykämien von 16,6 Ereignissen pro Patient und Jahr bei alleiniger Insulintherapie auf 21,6 Ereignisse pro Patient und Jahr bei zusätzlicher Gabe des GLP-1-Analogons. Und auch die Rate an Hyperglykämien mit Ketoazidose stieg von 0,1 Ereignissen pro Patient und Jahr auf 0,5 [1, 2]. Daraufhin wurde vonseiten des Herstellers die Entwicklung dieses Therapieprinzips bei Typ 1-Diabetikern nicht mehr weiter verfolgt. „Trotzdem sollte bei übergewichtigen Typ 1-Diabetikern mit Insulinresistenz die Gabe eines GLP-1Analogons als off label use durchaus erwogen werden, zumal die Verträglichkeit gut ist", so Bergis. Jedoch müsse man das gesteigerte Hypoglykämierisiko im Auge behalten.

Dr. med. Peter Stiefelhagen

Literatur:

1. Mathieu et al., Diabetes Care 2016 Oct, 39(10): 1702-10

2. Ahren et al., Diabetes Care 2016 Oct, 39(10): 1693-701

Quelle: Herbstkongress der Deutschen Diabetes Gesellschaft, 11.11.2016 in Nürnberg 